\title{
MeL: Modelo de adaptación dinámica del proceso de aprendizaje en eLearning
}

\author{
1 Departamento de Informática. Universidad de Oviedo (España). \\ 2 Departamento de Psicología. Universidad de Oviedo (España). \\ 3 Chancellery (Learning and Teaching), Murdoch University (Australia)
}

Miguel Sanchez-Santillan ${ }^{1}$, MPuerto Paule-Ruiz ${ }^{1}$, Rebeca Cerezo², Víctor Alvarez-García ${ }^{3}$

\begin{abstract}
Resumen: En nuestra vida diaria hemos integrado progresivamente el uso de Internet. Esta incorporación también se ha producido en todos los niveles educativos, donde los entornos virtuales de aprendizaje son el medio utilizado, por profesores, estudiantes e instituciones, para el manejo y la distribución de experiencias educativas. Sin embargo, tal y como están diseñados estos sistemas, hacen que los estudiantes tengan dificultades para desplegar sus habilidades metacognitivas, además de provocar una sobrecarga cognitiva debido a una mala organización de los contenidos y de la navegación. Es necesario, por tanto, incluir en las plataformas de aprendizaje un mecanismo que permita la adaptación de estos sistemas a las características, necesidades y contexto del estudiante con el objetivo de optimizar el proceso de enseñanza-aprendizaje. En este trabajo se describe un modelo de adaptación para Learning Management Systems (LMSs) que utilizando variables centrales en el proceso de aprendizaje permite aplicar reglas adaptativas a los distintos tipos de contenidos y conocimientos que se han de transmitir o adquirir. A nivel aplicado, el modelo obtenido permite desarrollar cursos adaptados que dan soporte y promueven el aprendizaje y la autorregulación dentro de los entornos de aprendizaje virtuales.

Palabras clave: E-learning; hipermedia adaptativa; aprendizaje autorregulado; entornos virtuales de aprendizaje; educación superior.
\end{abstract} lado, entornos virtuales de aprendizaje, educación superior.

\section{Introducción}

Las tecnologías de la información y la comunicación (TICs) se han ido incorporando de manera progresiva en nuestra vida diaria y la educación ha sido un área donde la influencia de las TICs ha quedado claramente reflejada. En este sentido, el EEES (Espacio Europeo de Educación Superior) plantea un escenario marcado por el uso de nuevas tecnologías, pero no podemos considerar este factor como una exigencia o demanda añadida al alumno, sino más bien como un ajuste de la educación superior a su realidad más próxima.

El e-learning, b-learning y el uso de LMSs (Dagger, O'Connor, Lawless, Walsh, y Wade, 2007) es ya una realidad que está presente en los procesos de enseñanza-aprendizaje (E-A) de la educación superior (Morueta, Rodríguez, y Gómez, 2011). Cada vez son más las universidades que ofertan estudios exclusivamente online, y el uso de plataformas de gestión de contenidos educativos como soporte curricular es ya generalizado (García, Ruiz, Van Dort, y Pérez, 2010).

En un LMS, el alumno tiene un papel activo que no puede eludir, ya que ha de interactuar con el sistema si quiere aprender, a diferencia de la enseñanza tradicional, donde el alumno podría adoptar un papel pasivo, siendo el profesor el agente activo del proceso (Area-Moreira, 2000). Si bien hay muchas ventajas en el uso de entornos hipermedia de aprendizaje, también hay inconvenientes que se deben con-

* Dirección para correspondencia [Correspondence address]:

Miguel Sánchez Santillán. Universidad de Oviedo. Facultad de Psicología. Plaza Feijoó S/N- Cabina 1. 33003 Oviedo (España). E-mail: $\underline{\text { mel- }}$ sanchezsantillan@gmail.com
Title: MeL: a dynamic adaptive model of the learning process in eLearning. Abstract: The use of the Internet has been progressively integrated into our daily lives. This has also been true for all levels of education, where virtual learning environments have been the means by which teachers, students and educational institutions managed and distributed educational experiences. However, the present design of these systems make students have difficulties in deploying their meta-cognitive skills, in addition to producing a cognitive overload due to an inadequate content organization and navigation. Thus, it is necessary to provide learning platforms with a process that allows for the adaptation of these systems to students' characteristics, needs and context in order to enhance the teaching-learning process. This paper describes an adaptive model for Learning Management Systems (LMSs) that using variables central to the learning process allows for the application of adaptive rules to the different types of contents and knowledge to be transferred and acquired. In practice, the resulting model allows to develop adaptive courses that support and promote learning and self-regulation in virtual learning environments.

Key words: E-learning; adaptive hypermedia; self regulated learning; learning management systems; higher education. siderar. Entre ellos, problemas de usabilidad tales como: desorientación, distracción y sobrecarga cognitiva (Scheiter y Gerjets, 2007). En ocasiones, los alumnos no saben dónde se encuentran dentro de la plataforma, a dónde ir, o cómo llegar a donde quieren; cuando se manejan demasiados temas relevantes en paralelo, esto puede distraer la atención de la tarea principal; y la comprensión de un problema o la toma de decisiones, puede verse interferida por la presencia de demasiada información. Estos problemas, como la sobrecarga de información, están motivados en gran parte por una mala organización de los contenidos y una navegación poco eficiente (Ruiz, Díaz, Soler, y Pérez, 2008), y podrían provocar el abandono de la plataforma y por tanto del proceso de aprendizaje por parte del estudiante. Además, variables como los conocimientos previos del alumno y los estilos y actitudes hacia el aprendizaje, parecen ser condicionantes y particularmente determinantes del proceso de aprendizaje en contextos hipermedia (Greene, Costa, Robertson, Pan, y Deekens, 2010; Moos y Azevedo, 2008).

En esta misma línea, aunque las virtudes en términos motivacionales del uso de TICs en el aprendizaje parece ser una hipótesis ya confirmada, recientes investigaciones muestran que estudiantes de todas las edades tienen dificultades para desplegar sus habilidades metacognitivas, las cuales juegan un rol fundamental en el aprendizaje, cuando éste se lleva a cabo en contextos abiertos, como los LMSs o los Computer Based Learning Environments (CBLEs) (Azevedo, Behnagh, Duffy, Harley, y Trevors, 2012; Azevedo, Cromley, Moos, Greene, y Winters, 2011; Azevedo, 2005). Estos entornos, aun no garantizando que el aprendizaje sea no lineal, propician y posibilitan que el alumno actúe como un 
agente activo en el proceso que sigue para aprender, es decir, puede decidir qué contenidos y rutas de navegación sigue para llegar hasta los resultados de aprendizaje de una manera no-lineal.

Aprender en CBLEs o en un LMS requiere un esfuerzo por parte del alumno a la hora de decidir qué va a aprender, cómo y cuánto, si se siente capaz o no, cuándo aumentar o ahorrar esfuerzos, etc. (Azevedo, Cromley, Winters, Moos, y Greene, 2005). Muchos de estos interrogantes quedan con frecuencia resueltos durante una clase presencial; el profesor transmite unos contenidos concretos, en un lugar concreto, durante un tiempo concreto, se realizan las tareas que indica el profesor, éste puede realizar apreciaciones acerca de la complejidad de esa clase que servirían para guiar la inversión de tiempo y esfuerzo de los alumnos, además de otras conductas de andamiaje que, tal como se recoge en el trabajo de McLoughlin del 2000, marcarían la diferencia en el proceso de aprendizaje que tiene lugar presencialmente respecto del no presencial, en este caso e-learning. En este sentido, los datos de los que se dispone respecto al aprendizaje en educación superior indican que la mayoría de los estudiantes no están adecuadamente preparados para las exigencias de estos contextos de aprendizaje (Azevedo y Feyzi-Behnagh, 2011; Azevedo y Aleven, 2013).

Desde la ciencia cognitiva, la psicología, e incluso desde la inteligencia artificial, se han obtenido resultados que nos muestran que los entornos de aprendizaje con soporte virtual suponen dificultades añadidas al estudiante en disciplinas fundamentales como las ciencias, las matemáticas o las ciencias sociales (Azevedo, 2005). Se han obtenido resultados empíricos convergentes que avalan la idea de que estas dificultades se deben a que éstos no despliegan procesos autorregulatorios claves durante su aprendizaje (Azevedo et al., 2011; Azevedo y Witherspoon, 2009; Lehmann, Hahnlein, y Ifenthale, D., 2014; Quintana, Zhang y Krajcik, 2005), entre los cuales se encuentran componentes motivacionales como la percepción de autoeficacia y el tipo de meta que guía el proceso de estudio. La motivación y la retroalimentación juegan un papel crucial en el aprendizaje y constituyen un área fundamental en todas las aproximaciones al aprendizaje autorregulado (SRL - self regulated learning) (Butler y Winne, 1995; Valle et al., 2010). Los estudiantes autorregulados se caracterizan por creencias motivacionales y actitudes adaptativas, como por ejemplo altos niveles de autoeficacia o competencia percibida y orientación a metas de aprendizaje (Paul R. Pintrich, 2000; Schunk y Ertmer, 2000). Asimismo, los alumnos juegan un rol activo en la generación y uso del feedback, lo cual conlleva implicaciones profundas en la manera en la que se promociona y evalúa el aprendizaje en soportes virtuales (Nicol y Macfarlane-Dick, 2006).

En este contexto, emerge el estudio de los entornos virtuales de aprendizaje adaptados al estudiante, una línea de investigación relativamente reciente que ha generado, sobre todo, conocimiento a nivel básico y no tanto aplicado (Tinajero, Castelo, Guisande, y Páramo, 2011; Vargas, Martínez, y Uribe, 2012). La investigación sobre hipermedia educacional adaptativa surge en la década de los 90, con la idea de crear sistemas educacionales adaptados a los estudiantes. Las primeras propuestas de investigación aparecen entre 1990 y 1996, dando lugar a dos líneas: los Tutores inteligentes (ITS) y los Sistemas hipermedia educacionales (SHE).

Los sistemas tutores inteligentes utilizaban técnicas de inteligencia artificial para representar el conocimiento e interactuar con los alumnos (Polson y Richardson, 1988). Estaban orientados al profesor y combinaban materiales de estudio con cuestionarios. La adaptación se fundamenta en una secuenciación del curso dependiendo del resultado de cuestionarios que realizaban los alumnos.

Los SHE están basados en la hipermedia adaptativa (Brusilovsky, 1996) con el objetivo de adaptar el sistema a las necesidades y características del alumno (estilos de aprendizaje, conocimientos, intereses, objetivos, etc). Están orientados al estudiante y son más una guía en el proceso de E-A que una secuenciación de contenidos a completar. La adaptación en los SHE es implementada empleando una o varios de las siguientes técnicas:

- Adaptación condicionada a que el alumno cumpla una serie de requisitos. Ejemplo de este tipo de sistemas son AHA! (Bra y Calvi, 1998) y My Online Teacher (Cristea y De Mooij, 2003).

- Contenidos didácticos con un formato particular y que tienen incluida la adaptación a un determinado contexto. AuldLinky (Michaelides, Millard, Weal, y DeRoure, 2002) es un sistema que pertenece a este segundo tipo.

- A través del empleo de lenguajes de alto nivel que el profesor ha de aprender para incluir la adaptación (Cristea, Smits, y Bra, 2007).

- Algoritmos de minería de datos para procesar la interacción del alumno con la plataforma proporcionándole información adaptada (Romero, Ventura, y García, 2008; Romero, Ventura, Zafra, y Bra, 2009).

Por otro lado, la aparición de la Web tuvo un gran impacto en los sistemas educacionales, propiciando la aparición de los entornos virtuales de aprendizaje, cuya evolución está más determinada por las tendencias tecnológicas que por la adecuación de las estrategias pedagógicas. Por tanto, estas plataformas, al contrario que los SHE, carecían de procedimientos de adaptación maduros (Hauger y Köck, 2007). En la actualidad, algunos LMSs ya introducen mecanismos de adaptación (Akbulut y Cardak, 2012). Es el caso de Moodle, que ha implementado procesos basados tanto en la interacción del alumno con la plataforma como en su perfil.

Estos entornos emplean un modelo de usuario estático, donde las variables están predefinidas y el valor asignado a éstas, para un determinado alumno, es común en todos los cursos del LMS. Este enfoque, poco flexible, imposibilita un mecanismo de adaptación dinámico que permita adecuarse a cada situación concreta de aprendizaje y supone un gran reto para la investigación actual (Bouchet, Harley, Trevors y Azevedo, 2013; Shute y Zapata-Rivera, 2012). 
En este escenario, el objetivo del trabajo es superar alguna de las dificultades añadidas que puede suponer para el estudiante aprender en entornos hipermedia virtuales de aprendizaje. Para ello, este trabajo plantea un modelo de adaptación del entorno de aprendizaje teniendo en cuenta la importancia y el valor potencial de los factores motivacionales. Como ejemplo de aplicación, hemos seleccionado las variables autoeficacia y metas de aprendizaje para adaptar uno de los LMSs más usados a las particularidades de cada alumno, de modo que se puedan salvar algunas de las dificultades ya planteadas, como el abandono de la tarea por una baja percepción de autoeficacia, o una mala elección de las metas que guiarán el aprendizaje.

Hasta esta década, la mayoría de los trabajos acerca del aprendizaje en entornos hipermedia se han centrado sobre todo en variables cognitivas, siendo mucho menor la atención prestada a las variables de naturaleza motivacional (Lepper y Woolverton 2004; Moos y Azevedo, 2009; Zimmerman y Tsikalas 2005). Sin embargo, ya comenzaron a identificarse las variables autoestima, autoeficacia y motivación, como particularmente importantes para predecir el éxito académico del proceso de e-learning en 1998, con el trabajo de Gibson, o de Pachnowski y Jurczyk en el 2000. Estos resultados no resultan sorprendentes ya que, en general, el estudiante que aprende a distancia tiene menor contacto tanto con el profesor como con sus compañeros, por tanto deberá confiar en mayor medida en sus estrategias de estudio (Moore, 1989). Más recientemente, el peso de estas variables en e-learning respecto de formatos presenciales se ha ido confirmando con trabajos como los de Deka y McMurry en 2006 o los de Hsu y Huang en el mismo año.
En concreto, autoeficacia y metas de estudio, parecen ser dos de las variables cruciales a la hora de estudiar la vertiente motivacional del proceso de aprendizaje en CBLEs. Tanto es así que se ha hecho necesario reformular constructos clásicos como la autoeficacia en otros más concretos como computer self-efficacy (Murphy et al., 1989), o las metas de aprendizaje dentro del modelo Regulating Motivation and Performance Online, y estudiarlos como determinantes del aprendizaje que tiene lugar en CBLEs (Hsu y Huang, 2006; Johnson, 2005; Matt, 2014; Moos y Azevedo, 2009; Pellas, 2014; Sansone, Fraughton, Zachary, Butner, Heiner, 2011).

\section{Modelo teórico de adaptación para sistemas de ges- tión de aprendizaje}

Tal como se ha planteado, este trabajo propone un modelo teórico de adaptación cuyo objetivo es apoyar el proceso de E-A, considerando la influencia en el aprendizaje de parámetros dinámicos, no deterministas, que dependen del contexto, características y necesidades del estudiante. El modelo de adaptación propuesto tiene en cuenta los siguientes requisitos básicos, que al mismo tiempo delimitan los agentes implicados en el proceso (Figura 1):

- Permitir a los docentes adaptar los cursos del LMS a las características del alumno, mediante un modelo de usuario flexible, basado en el contexto de aprendizaje.

- Proporcionar mecanismos de retroalimentación adaptados a las necesidades del alumno y que permitan tanto a los docentes como a los alumnos, realizar un seguimiento del progreso y estado del aprendizaje, facilitando así la toma de decisiones en el proceso de E-A.
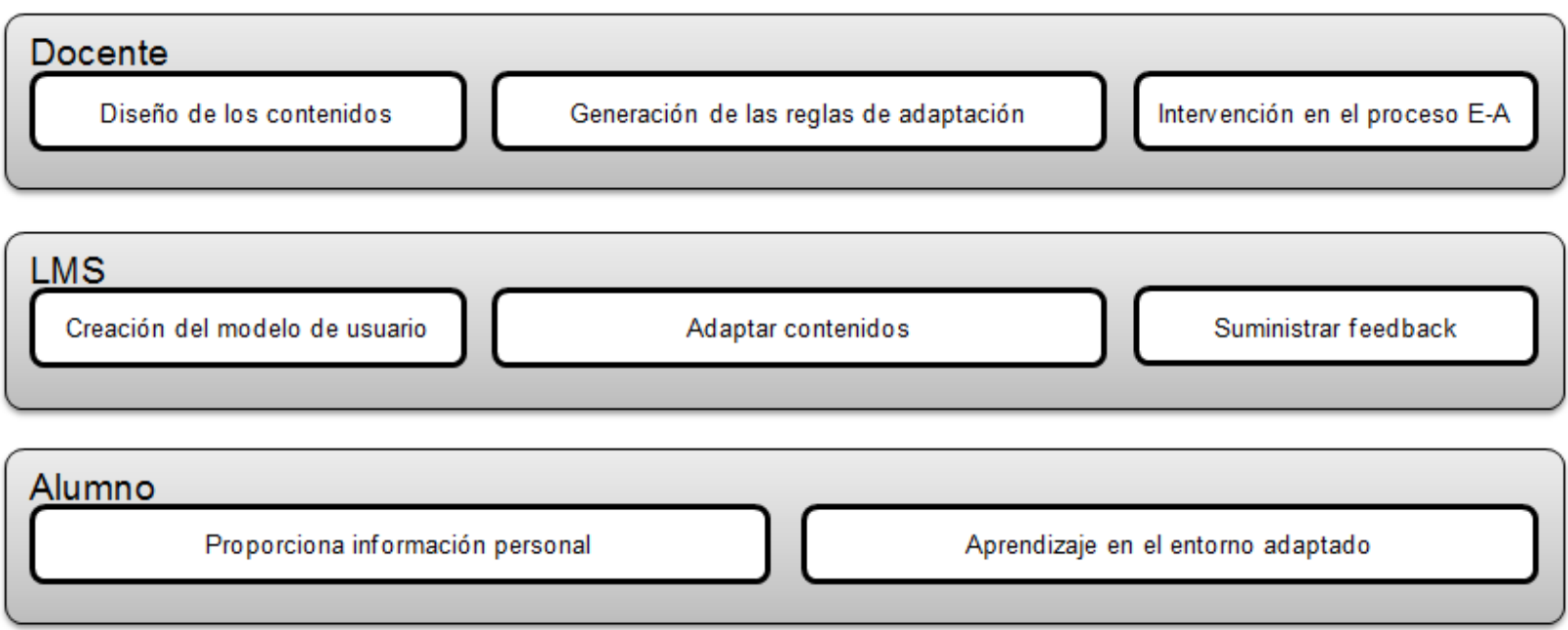

Figura 1. Identificación de los agentes implicados en el modelo dinámico de adaptación y acciones que llevan a cabo.

Por tanto, es necesario realizar una clasificación genérica de las variables que moldean al alumno para que el docente pueda contribuir a la adaptación. El LMS realiza el proceso de adaptación y proporciona la retroalimentación.

\section{Naturaleza de las variables de adaptación}

Si bien trabajos previos proponen realizar una adaptación en base a un conjunto predeterminado de variables, nuestra herramienta permite a los docentes seleccionar las 
variables que describen las características, necesidades y contexto de los alumnos, y definir el proceso de adaptación de los contenidos del curso.

En este modelo diferenciamos entre tres tipos de contenidos que se enseñan o aprenden a través del LMS: teóricos, prácticos y aplicados. Del mismo modo que el proceso de EA difiere en función de sus características, las variables que modulan el proceso de aprendizaje de cada uno de los tipos de contenido, también variarán. De este modo, se ha de diferenciar la adaptación de contenidos puramente teóricos, frente a la adaptación de la puesta en práctica de esos conocimientos. Esta circunstancia se sustenta sobre la diferenciación clásica de los tres tipos de conocimiento: declarativo o conceptual, procedimental y condicional (Brown, 1987; Jacobs y Paris, 1987; Schraw y Moshman, 1995). Por ello, el modelo emplea la siguiente clasificación de variables:

- Variables macro contextuales: son aquellas variables ajenas a la plataforma virtual y que funcionan tanto si la situación de aprendizaje se realiza en una clase magistral como a través de una herramienta de e-learning. Diferenciando entre:

- Variables de rasgo: no están bajo el control del alumno pero influyen en su aprendizaje. E.g., género, edad, etc.

- Variables de estado: dependen de la situación concreta del estudiante en un determinado escenario de aprendizaje. E.g., creencias de autoeficacia, expectativas de logro, metas académicas, etc.

- Variables micro contextuales: dependientes de la plataforma o de la situación de aprendizaje virtual concreta. E.j., detalle de visualización de un curso, representación del feedback, estilos de visualización, etc.

\section{Arquitectura de la adaptación y del Feedback}

El modelo de adaptación y de feedback está compuesto de cuatro capas (Figura 2): capa de instrumentos, capa de variables contextuales, capa de procesos de adaptación y capa de retroalimentación.

\section{Capa de instrumentos de evaluación}

Es la encargada de recopilar la información que permite definir variables dentro del LMS para ser empleadas en la adaptación. Según la naturaleza del instrumento, se clasifica en una de las siguientes categorías:

- Test: autoinformes adaptados a entornos de aprendizaje virtuales validados y extensamente usados por la comunidad científica.

- Históricos: conjunto de datos relacionados con los alumnos, ya sea la información disponible en las plataformas, o los ficheros de logs que registran la interacción del estudiante.

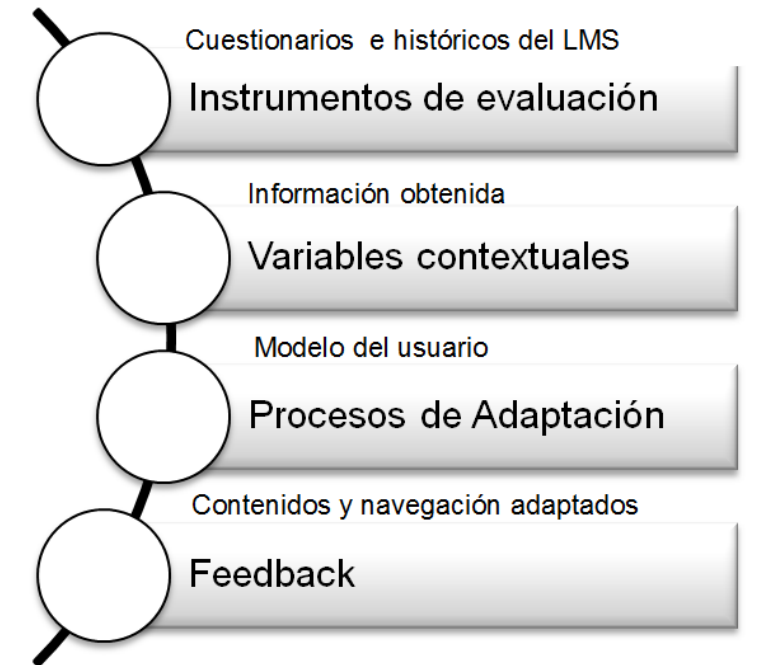

Figura 2. Modelo de capas: relación entre cada una de las capas en función de la secuencia y los datos que comparten.

\section{Capa de variables contextuales}

Procesa y clasifica la información generada a través de los instrumentos. Su funcionamiento se realiza en dos fases:

- Fase de procesado de variables: las variables definidas en los instrumentos son incorporadas al sistema de manera automática. En algunos casos, el docente debe de intervenir, ajustando la asignación de valores a la muestra.

- Fase de procesado de resultados: es la fase encargada de analizar los resultados recogidos por los instrumentos para construir el modelo de usuario.

Estas dos fases proporcionan al LMS un modelo de usuario flexible, ya que el conjunto de variables y sus valores están determinados por el contexto y la posible intervención del docente.

\section{Capa de procesos de adaptación}

La capa de procesos de adaptación es la responsable de gestionar y aplicar las reglas descritas por los docentes para adaptar el contenido y navegación del curso al alumno. Las reglas son de tipo condicional y están compuestas de:

- Precondición: el conjunto de valores-variables que se deben cumplir para que se activen las acciones de una regla.

- Acciones: las acciones son las encargadas de realizar la adaptación, tanto de los contenidos como de la navegación en el LMS. En su dimensión técnica, las acciones están basadas en la aplicación de los métodos y técnicas de la hipermedia adaptativa (Brusilovsky, 1996).

Las reglas, según su objetivo, se clasifican en una de las siguientes categorías:

- Reglas de disponibilidad: indican cómo un alumno interactúa con los contenidos de un curso. Estas reglas de- 
terminan si para un alumno un contenido es: (a) accesible, (b) visible pero no accesible o (c) no accesible.

- Reglas de completitud: indican qué acciones se desencadenan cuando se completa un contenido de la plataforma. Ejemplos de acciones son: mostrar un contenido personalizado, enviar un mensaje o cambiar el valor de una variable contextual.

Las reglas de disponibilidad y completitud están interrelacionadas. Completar un contenido puede, a su vez, activar una regla de disponibilidad. Esta reacción en cadena, permite a los docentes establecer diferentes guías de navegación adaptadas a los alumnos.

\section{Capa de feedback}

Analiza la interacción del alumno con el LMS y proporciona información al alumno y al profesor sobre el progreso en el entorno de aprendizaje. Se aplican dos niveles de retroalimentación:

- Docente-sistema-alumno: tiene dos componentes:
- La retroalimentación que el docente proporciona al alumno acerca de sus resultados como complemento a la evaluación de las tareas.

- La retroalimentación basada en las reglas de completitud, creadas por el docente y activadas por el sistema, para proporcionar al alumno indicadores de aprendizaje, tal y como propone Duval (2011).

- Alumno-sistema-docente: el sistema analiza la interacción del alumno con los contenidos del curso y proporciona al docente informes y gráficos del progreso de los estudiantes.

Esta información facilita al docente la toma de decisiones, ayuda a los estudiantes a monitorizar sus logros, y orienta y estimula la reflexión en el proceso de aprendizaje y las competencias adquiridas (Glahn, Specht, y Koper, 2007).

Una vez concretadas las variables, capas, agentes y elementos que posibilitan la adaptación, en la Figura 3 se representa la interacción de los agentes con la arquitectura del modelo.

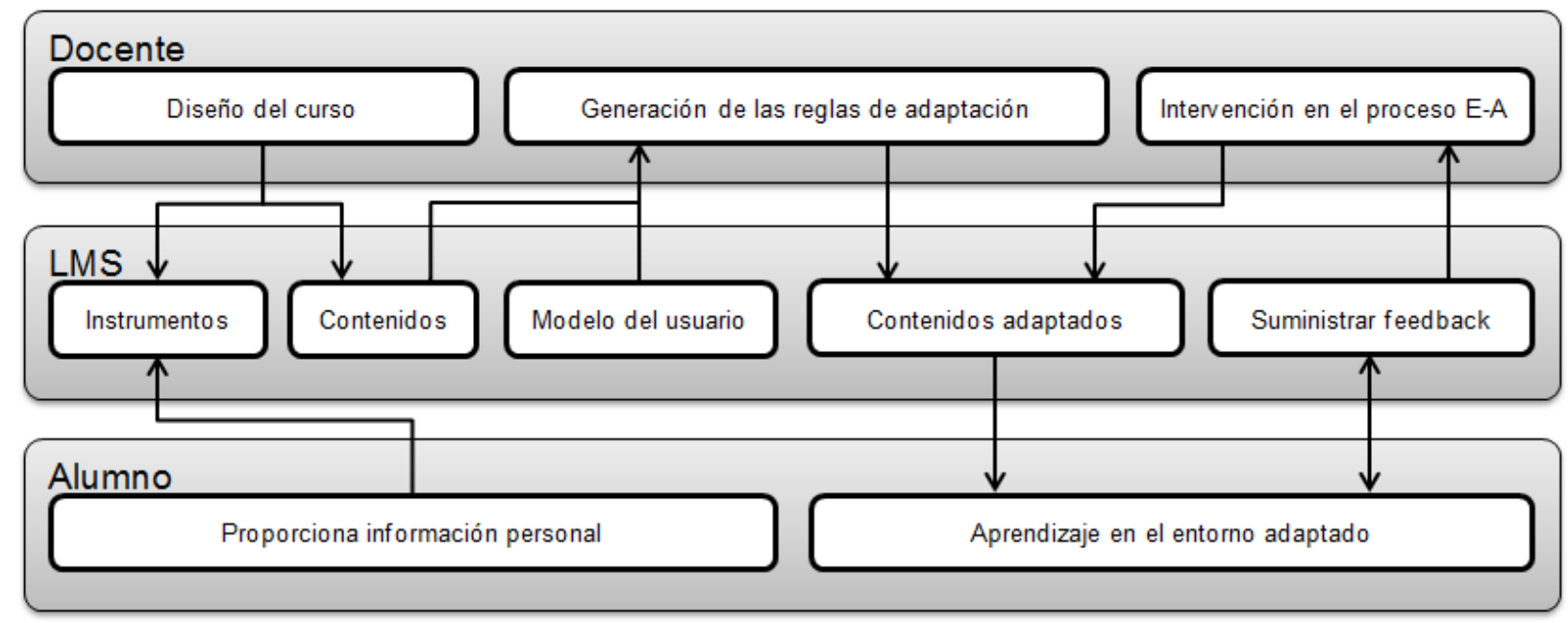

Figura 3. Interacción de los agentes implicados con la arquitectura del modelo.

\section{Aplicación del modelo teórico a variables motiva- cionales}

En este trabajo se propone un modelo flexible y general, de manera que sea aplicable a diferentes LMSs y útil para cualquier nivel educativo. Sin embargo, resulta especialmente adecuado para educación superior, donde los entornos virtuales de aprendizaje han sido implantados con éxito y los alumnos tienen mayor autonomía en su aprendizaje. A continuación, se describe una aplicación del proceso de adaptación de cara a mejorar la comprensión del modelo. Para ello se han tenido en cuenta contenidos reales no adaptados de una asignatura del plan de estudios del grado de Psicología. Una de las competencias de la asignatura es que los alumnos aprendan a trabajar de forma autorregulada y entrenen estrategias de aprendizaje y estudio interaccionando con los con- tenidos teóricos y prácticos disponibles a través del LMS. Empleando el modelo propuesto, se adaptan dichos contenidos en función de la percepción de autoeficacia y el tipo de meta que guía el proceso de aprendizaje de cada alumno.

\section{Instrumentos y variables}

Las variables de interés en función de las cuales se adapta el entorno al alumno son Autoeficacia Percibida y Metas de Estudio (Tabla 1).

La Autoeficacia Percibida, a su vez, tendría dos niveles, macro y micro, atendiendo a las diferencias encontradas en la literatura entre General Self-efficacy y Specific Self-efficacy (Agarwal, Sambamurthy, y Stair, 2000; Schunk, 1995). 
Tabla 1 Instrumentos de medida y posibles valores que pueden tomar las variables de adaptación

\begin{tabular}{lll}
\hline Instrumento & Variable & Posibles valores \\
\hline MSLQ & Autoeficacia macro & Baja - Media - Alta \\
MSLQ & Metas de Estudio & $\begin{array}{l}\text { Logro }- \text { Aprendizaje - } \\
\text { Combinada }\end{array}$ \\
$\begin{array}{l}\text { Formulario } \\
\text { Histórico del LMS }\end{array}$ & $\begin{array}{l}\text { Autoeficacia micro } \\
\text { Contenido/tarea } \\
\text { completada }\end{array}$ & $\begin{array}{l}\text { Baja - Media - Alta } \\
\text { Completado - No com- } \\
\text { pletado }\end{array}$ \\
\hline
\end{tabular}

- Autoeficacia Macro: relativa a cuán eficaces se perciben los sujetos, en general, para aprender en el entorno virtual. Esta variable se mide a través de la subescala de creencias de autoeficacia del MSLQ - Motivated Strategies for Learning Questionnaire (Pintrich, Smith, Garcia, y McKeachie, 1991), compuesta por 8 ítems a los que el sujeto debe dar una puntuación de 1 a 7 en función del ajuste a su situación personal. La formulación de los ítems ha sido ligeramente modificada para ajustarlos a las peculiaridades del entorno virtual tal como se ha hecho con anterioridad en la literatura (Moos y Azevedo, 2008).

- Autoeficacia Micro: relativa a cuán eficaces se perciben los sujetos, en particular, para enfrentarse a determinados contenidos o tareas. Esta variable se evalúa a través de un único ítem que pregunta al sujeto cómo de eficaz se percibe para afrontar unos contenidos específico del tema o una tarea concreta en una escala de 1 a 7.

La variable Metas de Estudio, asimismo, tendría dos niveles, Orientación a Metas Extrínseca y Orientación a Metas Intrínseca (Valle et al., 2003, 2009). En función de ella se clasifica a los sujetos a través de la subescala de componente de valor del MSLQ (Pintrich et al., 1991).

- Orientación a Metas Extrínseca: este tipo de metas se describen como aquellas que llevan al individuo a realizar una determinada acción para la consecución de otros motivos no relacionados con la actividad, sino con la consecución de otras metas que en el campo académico suelen ser: obtener buenas notas o recompensas materiales, lograr reconocimiento social, evitar el fracaso, etc.

- Orientación a Metas Intrínseca: se vincula este tipo de metas a aquellas acciones realizadas por el interés que genera la propia actividad, no como un medio para alcanzar otras metas.

La variable Metas de Estudio es medida a través de 8 ítems (4 correspondientes a Metas Extrínsecas y otros 4 para Metas Intrínseca) a los que el sujeto debe dar una puntuación de 1 a 7 en función del grado de ajuste a su situación personal. La formulación de los ítems también ha sido ligeramente modificada para ajustarse a las peculiaridades de los entornos virtuales.

\section{Ejemplo de adaptación}

En la Figura 4, se muestra el proceso de adaptación aplicado a los contenidos de la asignatura. Según el valor que tomen las variables, la interacción del alumno con los contenidos (teóricos/prácticos) varía, ofreciéndole la posibilidad de elegir diferentes modalidades de contenido o guiándole a través de una secuencia determinada.

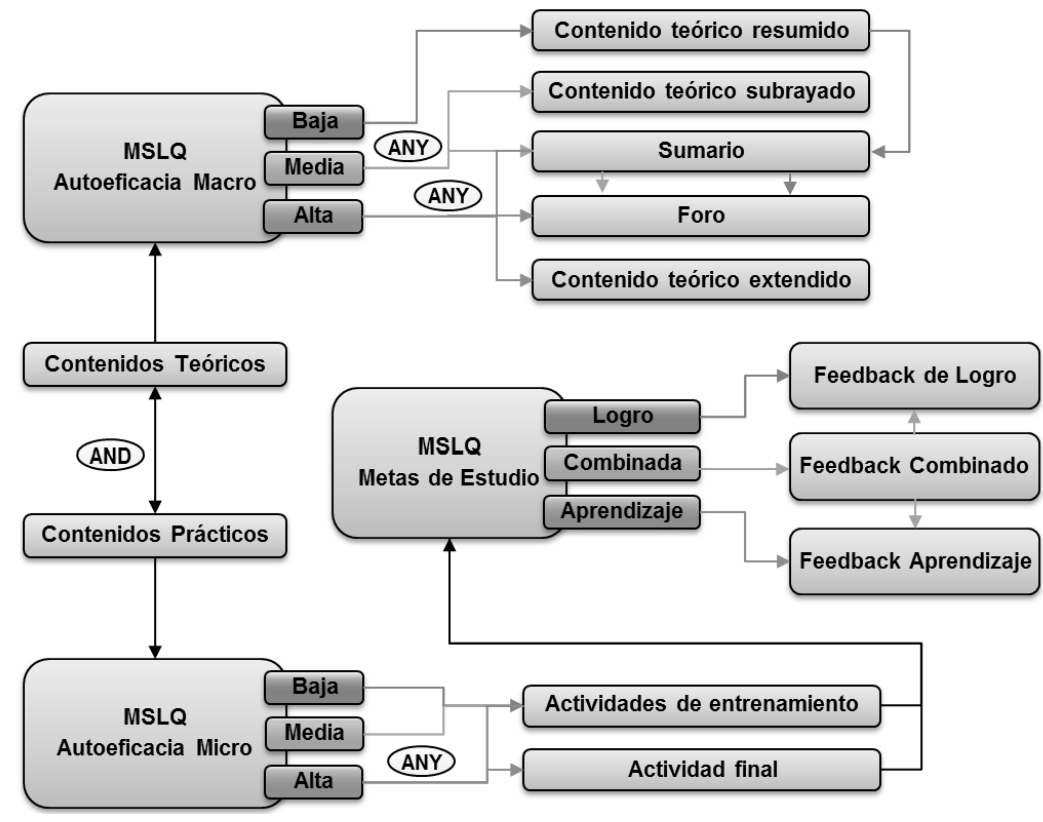

Figura 4. Representación de la adaptación y feedback en el caso propuesto.

Para comprender el ejemplo, es necesario conocer previamente los elementos susceptibles de adaptación que con- forman la asignatura. Por un lado, los contenidos puramente teóricos con tres niveles de complejidad: contenido teórico 
extendido, contenido teórico subrayado y contenido teórico resumido. Además, se cuenta con sumarios de cada una de las unidades de contenido con la información indispensable para el alumno. Por otro lado, los contenidos aplicados a la práctica; para ello se cuenta con un banco de tareas que iteran en función de su grado de complejidad y de los resultados del alumno. Finalmente, el curso se completa con recursos alternativos como foros, audios, vídeos, etc. que se incluyen en cada tema como complemento de los contenidos teórico-prácticos.

Una vez delimitados los elementos disponibles en el curso, el LMS calcula las posibles rutas adaptativas que puede seguir un alumno dentro de esta asignatura. Por ejemplo, si el alumno se enfrenta al contenido teórico, y su valor en la "autoeficacia macro" es "bajo", las reglas de disponibilidad obligan a que complete los contenidos en el siguiente orden: "contenido teórico resumido" y "sumario del tema". Sin embargo, si el valor de la "autoeficacia macro" es "medio", el alumno elige qué contenido completar primero, "contenido teórico subrayado" o bien "sumario del tema". Por último, si la "autoeficacia macro" es "alta", se le añade la posibilidad de acceder al "contenido teórico extendido". Solo cuando el alumno complete los contenidos teóricos se le redirige al foro para que pueda participar en él.

En el caso de las tareas, la adaptación se lleva a cabo en base a la variable Autoeficacia Micro. En esta fase se pretende asegurar, al menos, algún éxito inicial a todos los estudiantes, especialmente a aquellos que parten de un nivel de autoeficacia micro bajo. Todos los alumnos, independientemente de que partan de un nivel alto, bajo o alto, deben de finalizar la fase de resolución de tareas con un nivel alto, es decir, con la percepción de que pueden resolver la tarea planteada eficazmente y demostrar que así lo hacen. Para ello, el LMS combina las puntuaciones en los ítems de autoeficacia micro y los resultados en cada tarea concreta, y calcula en tiempo real la ruta para la resolución de las tareas prácticas en función del nivel de complejidad más adecuado.

\section{Adaptación del feedback}

Se realiza a dos niveles:

- Docente-sistema-alumno: el alumno recibe mensajes personalizados donde se le indica el progreso de su aprendizaje, así como las competencias adquiridas. El contenido de estos mensajes, está adaptado según la variable Metas de Estudio. Por ejemplo, si el alumno obtiene una puntuación que le sitúa en metas de aprendizaje, se le retroalimenta reforzando las competencias que ha adquirido al superar un determinado contenido (e.g. muy bien, estás más cerca de conocer cómo funciona nuestra memoria; enhorabuena, parece que ya sabes cómo hacer mapas conceptuales). Si por el contrario es un estudiante orientado a metas de logro, la retroalimentación resalta la puntuación obtenida al superar ese contenido (e.g. muy bien, estás más cerca de completar la evaluación final; enhorabuena, has obtenido una puntuación de 0.8 sobre 1; respuesta correcta, sigue contestando y obtendrás más puntos; respuesta incorrecta, sigue contestando para poder obtener más puntos). A aquellos alumnos que se guían por metas combinadas se les da un feedback también combinado de aprendizaje y logro. La retroalimentación de los sujetos con metas de logro o con metas de aprendizaje se intercala también con feedback combinado en consonancia a los estudios sobre metas, en los que se observa que una combinación de ambas parece ser la orientación que optimiza los resultados de aprendizaje (Rodríguez et al., 2001; Valle et al., 2003, 2009).

- Alumno-sistema-docente: a través de informes y gráficos, el docente dispone de la información acerca del progreso individual y colectivo de los alumnos en cada tema (Duval et al., 2012). La información incluye los contenidos visitados, las actividades realizadas, los tiempos y periodos de actividad, el rendimiento, la variación de los valores en la autoeficacia y las metas, etc.; una amplia variedad de indicadores inter e intra sujeto que permiten al docente conocer el proceso de aprendizaje del alumno y no solo su producto. En concreto, tanto las herramientas propias del LMS para visualizar decenas de acciones personalizables en función de los intereses del docente (registros, registros activos, actividad del curso, vistas, participación en el curso, calificaciones, etc.) en diferentes formatos (mostrar en página, formato de texto, formato ODS, formato Excel, gráficos de visualización, etc.), como un plug-in especifico desarrollado adhoc para la implementación de MeL (véase eGraph en Cerezo, Álvarez, Sánchez-Santillán, Núñez, Álvarez, 2013).

Bajo nuestro punto de vista, la retroalimentación refuerza la monitorización y favorece la adaptación del proceso de E-A, permitiendo que el docente establezca nuevas estrategias instruccionales, y que el propio estudiante oriente el aprendizaje en consonancia a sus metas de estudio.

\section{Conclusiones}

En este estudio presentamos un modelo teórico de adaptación de los entornos de aprendizaje a los estudiantes. Este objetivo viene motivado principalmente por las dificultades añadidas que experimentan los estudiantes cuando aprenden en entornos de aprendizaje hipermedia, como los LMSs o los CBLEs (Azevedo et al., 2012, 2011; Scheiter y Gerjets, 2007).

El estudio está basado en tres premisas fundamentales: el alumno es sujeto activo y dinámico en el aprendizaje que tiene lugar en entornos virtuales, el profesor ha de poder andamiar y adaptar ese proceso de aprendizaje, las plataformas deben poder adaptar sus contenidos y navegación, y proporcionar feedback en tiempo real del proceso de E-A.

A partir de los supuestos anteriores, se ha desarrollado un modelo teórico de adaptación fundamentado en un modelo de usuario dinámico, un sistema de reglas diseñadas por el docente, y un proceso automático de adaptación y retro- 
alimentación que realiza el LMS. Tradicionalmente, el modelo de usuario, así como el proceso de adaptación en los sistemas, era estático y poco flexible. La alternativa propuesta, permite crear nuevas variables añadiendo instrumentos a la plataforma, posibilitando al docente emplear procesos de adaptación propuestos por otros autores, como por ejemplo: adaptación basada en estilos cognitivos y estilos de aprendizaje (Yang, Hwang, y Yang, 2013) o adaptación teniendo en cuenta las discapacidades y preferencias del alumno (Cuesta, Ramos, y Pavlich-Mariscal, 2012). Por tanto, el presente modelo permite a los docentes conocer y seleccionar las variables que delimitan las características, necesidades y contexto de los alumnos, y definir las reglas de adaptación. El LMS es el agente encargado de regular el proceso de adaptación a través de técnicas de Data Mining (Romero et al., 2008) e Hipermedia Adaptativa (Brusilovsky, 1996), además de ofrecer feedback sobre el proceso de aprendizaje tanto a los profesores como a los estudiantes (Duval, 2011).

Se ha sustentado el modelo con una arquitectura en la que se describen tanto los procesos como los datos que dan soporte a la adaptación. Además, se ha aplicado el modelo teórico a las variables motivacionales de autoeficacia y metas

\section{Referencias}

Agarwal, R., Sambamurthy, V., y Stair, R. M. (2000). Research Report: The Evolving Relationship Between General and Specific Computer SelfEfficacy-An Empirical Assessment. Information Systems Research, 11(4), pp. 418-430. doi:10.1287/isre.11.4.418.11876

Akbulut, Y., y Cardak, C. S. (2012). Adaptive educational hypermedia accommodating learning styles: A content analysis of publications from 2000 to 2011. Computers \& Education, 58(2), 835 - 842. doi:10.1016/j.compedu.2011.10.008

Area-Moreira, M. (2000). What is the role of the Internet in the pedagogical change at the level of College Education (Spanish). Proceedings of III International Conference of Communication, Technology and Education, pp. 128135.

Azevedo, R. (2005). Using Hypermedia as a Metacognitive Tool for Enhancing Student Learning? The Role of Self-Regulated Learning. Educational Psychologist, 40(4), 199-209. doi:10.1207/s15326985ep4004_2

Azevedo, R., \& Aleven, V. (Eds.). (2013). International handbook of metacognition and learning technologies. Amsterdam, The Netherlands: Springer

Azevedo, R., Behnagh, R., Duffy, M., Harley, J., y Trevors, G. (2012). Metacognition and self-regulated learning in student-centered leaning environments. D. Jonassen \& S. Land. Theoretical foundations of student-center learning environments, 171-197.

Azevedo, R., Cromley, J. G., Moos, D. C., Greene, J. A., y Winters, F. I. (2011). Adaptive content and process scaffolding: A key to facilitating students' self-regulated learning with hypermedia. Psychological Testing and Assessment Modeling, 53, 106-140

Azevedo, R., Cromley, J. G., Winters, F. I., Moos, D. C., y Greene, J. A. (2005). Adaptive Human Scaffolding Facilitates Adolescents' Selfregulated Learning with Hypermedia. Instructional Science, 33(5-6), 381412. doi:10.1007/s11251-005-1273-8

Azevedo, R., y Feyzi-Behnagh, R. (2011). Dysregulated Learning with Advanced Learning Technologies. Journal of e-Learning and Knowledge SocietyEnglish Version, 7(2).

Azevedo, R. y Witherspoon, A. M. (2009). Self-regulated learning with hypermedia. En D. J. Hacker, J. Dunlosky, and A. C. Graesser, editors, Handbook of metacognition in education, pp 319-339. Mahwah, NJ: Routledge.

Bouchet, JM Harley, GJ Trevors, \& R Azevedo (2013). Clustering and Profiling Students According to their Interactions with an Intelligent Tu- de estudio con el objetivo de permitir que los profesores diseñen un curso adaptado y que los alumnos desplieguen procesos autorregulatorios claves durante su aprendizaje (Azevedo et al., 2011; Azevedo y Witherspoon, 2009; Quintana, Zhang, y Krajcik, 2005).

En un futuro inmediato se plantea el desarrollo de estudios que nos permitan aplicar el modelo propuesto en otros entornos y diferentes cursos online. Para ello se ha desarrollado una herramienta a modo de plug-in que permite implementar este modelo y que actualmente ha superado los test de software satisfactoriamente.

Además, sería necesario evaluar el grado de completitud de los objetivos, es decir, determinar en qué medida se proporciona información descriptiva acerca de los alumnos y su proceso de aprendizaje, se facilita el diseño de cursos adaptados y, especialmente, se mejora el aprendizaje. Del mismo modo, se contempla la integración de variables de naturaleza cognitiva, así como profundizar en el estudio de las capas de procesos de adaptación y feedback. El resultado de combinar esta propuesta con otras técnicas adaptativas permitiría optimizar el ajuste de la adaptación dinámica para proporcionar una mejora en el proceso de E-A.

toring System Fostering Self-Regulated Learning. Journal of Educational Data Mining, 5(1), 104-146.

Bra, P. D., y Calvi, L. (1998). AHA! An open Adaptive Hypermedia Architecture. New Review of Hypermedia and Multimedia, 4, pp. 115-139. doi:10.1080/13614569808914698

Brown, A. (1987). Metacognition, executive control, self-regulation, and other more myster- ious mechanisms. En F. Weinert \& R. Kluwe, eds., Metacognition, Motivation, and Under-standing, (pp. 65-116. Hillsdale, NJ: Erlbaum.

Brusilovsky, P. (1996). Methods and techniques of adaptive hypermedia. User Modeling and User-Adapted Interaction, 6(2), 87-129. doi:10.1007/BF00143964

Butler, D. L., y Winne, P. H. (1995). Feedback and Self-Regulated Learning: A Theoretical Synthesis. Review of Educational Research, 65(3), 245-281. doi:10.3102/00346543065003245

Cerezo, R., Álvarez, D., Sánchez-Santillán, M., Núñez, J.C. y Álvarez, L. (2013). Nuevas metodologías para la evaluación del aprendizaje en campus virtuales. En O. Arias-Gundín \& R. Fidalgo (Eds.), Innovación Educativa en la Educación Superior (pp. 77-96). Saarbrücken: Lap Lamberte Academic Publishin GMBH \& Co.

Cristea, A. I., y De Mooij, A. (2003). Adaptive course authoring: My Online Teacher. En 10th International Conference on Telecommunications, 2003. ICT 2003 (Vol. 2, pp. 1762 - 1769 vol.2).

Cristea, A., Smits, D., y Bra, P. de. (2007). Towards a generic adaptive hypermedia platform: a conversion case study. JoDI: Journal of Digital Information, 8(3), 1.

Cuesta, D. L., Ramos, A. C., y Pavlich-Mariscal, J. A. (2012). Adaptation and disability aspects in a virtual learning environment. Dyna, 79(173), 6-14.

Dagger, D., O’Connor, A., Lawless, S., Walsh, E., y Wade, V. P. (2007). Service-Oriented E-Learning Platforms: From Monolithic Systems to Flexible Services. IEEE Internet Computing, 11(3), 28 -35. doi:10.1109/MIC.2007.70

Hauger, D., y Köck, M. (2007). State of the art of adaptivity in e-learning platforms. En Proceedings of the Workshop Adaptivity and User Modeling in Interactive Systems (ABIS) (pp. 24-26).

Duval, E. (2011). Attention please!: learning analytics for visualization and recommendation. En Proceedings of the 1st International Conference on Learning Analytics and Knowledge (pp. 9-17). New York, NY, USA: ACM. doi:10.1145/2090116.2090118 
Duval, E., Klerkx, J., Verbert, K., Nagel, T., Govaerts, S., Parra Chico, G. A., ... Vandeputte, B. (2012, May). Learning dashboards \& learnscapes. García, V. M. Á., Ruiz, M. del P. P., Van Dort, R., y Pérez, J. R. P. (2010). RSS-Based Learning Using Audio. International Journal of Human Capital and Information Technology Professionals, 1(4), 76-87. doi:10.4018/jhcitp.2010100105

Gibson, C. C. (1998). The distance learner's academic self-concept. En C.C. Gibson (Ed.) Distance Learners in Higher Education: Institutional responses for quality outcomes (p. 65-76). Madison, WI.: Atwood.

Glahn, C., Specht, M., y Koper, R. (2007). Smart Indicators on Learning Interactions. En E. Duval, R. Klamma, \& M. Wolpers (Eds.), Creating New Learning Experiences on a Global Scale (pp. 56-70). Springer Berlin Heidelberg.

Greene, J. A., Costa, L.-J., Robertson, J., Pan, Y., y Deekens, V. M. (2010). Exploring relations among college students' prior knowledge, implicit theories of intelligence, and self-regulated learning in a hypermedia environment. Computers \& Education, 55(3), 10271043.doi:10.1016/i.compedu.2010.04.013

Hsu, W. K., \& Huang, S. S. (2006). Determinants of computer self-efficacy: An examination of learning motivation and learning environments. Journal of Educational Computing Research, 35(3), 245-265.

Jacobs, J. E., y Paris, S. G. (1987). Children's Metacognition About Reading: issues in Definition, Measurement, and Instruction. Educational Psychologist, 22(3-4), 255-278. doi:10.1080/00461520.1987.9653052

Johnson, R. D. (2005). An empirical investigation of sources of applicationspecific computer self-efficacy and mediators of the efficacyperformance relationship. International Journal of Human-Computer Studies, 62(6), 737-758

Lehmann, T., Hähnlein, I. y Ifenthale, D. (2014) Cognitive, metacognitive and motivational perspectives on preflection in self-regulated online learning. Computers in Human Behavior, 32, 313-323.

Lepper, M., y Wolverton, M. (2004). The wisdom of practice: Lessons learned from the study of highly effective tutors. En J. Aranson (Ed.), Improving academic achievement: Impact of psychological factors on education (pp. 135-158). New York, NY: Academic Press.

Matt, H. (2014). Creation of a Computer Self-Efficacy Measure: Analysis of Internal Consistency, Psychometric Properties, and Validity. Cyberpsychology, Behavior, and Social Networking. doi:10.1089/cyber.2014.0255.

McLoughlin, C. (2000). Beyond the halo effect: Investigating the quality of student learning online. Proceedings Moving Online Conference, School of Social Sciences, Southern Cross University.

Michaelides, D., Millard, D., Weal, M., y DeRoure, D. (2002). Auld Leaky: A Contextual Open Hypermedia Link Server. En S. Reich, M. Tzagarakis, \& P. De Bra (Eds.), Hypermedia: Openness, Structural Awareness, and Adaptivity (Vol. 2266, pp. 215-218). Springer Berlin / Heidelberg.

Moos, D. C., y Azevedo, R. (2008). Self-regulated learning with hypermedia: The role of prior domain knowledge. Contemporary Educational Psychology, 33(2), 270-298. doi:10.1016/i.cedpsych.2007.03.001

Moos, D. C., y Azevedo, R. (2009). Learning With Computer-Based Learning Environments: A Literature Review of Computer Self-Efficacy. Review of Educational Research, 79(2), 576-600. doi: 10.3102/0034654308326083

Morueta, R. T., Rodríguez, M. A. P., y Gómez, J. I. A. (2011). Blended elearning en universidades andaluzas. Aula abierta, 39(2), 47-58.

Nicol, D. J., y Macfarlane-Dick, D. (2006). Formative assessment and self-regulated learning: a model and seven principles of good feedback practice. Studies in Higher Education, 31(2), 199-218. doi:10.1080/03075070600572090

Pellas, N. (2014). The influence of computer self-efficacy, metacognitive self-regulation and self-esteem on student engagement in online learning programs: Evidence from the virtual world of Second Life. Computers in Human Behavior, 35. 157-170. doi: 10.1016/j.chb.2014.02.048

Pachnowski, L. M., y Jurczyk, J. P. (2000). Correlating self-directed learning with distance learning success. Proceedings of the annual meeting of the Eastern Educational Research Association at Clearwater, FL.

Pintrich, P. R., Smith, D., Garcia, T., y McKeachie, W. J. (1991). A Manual for the Use of the Motivational Strategies for Learning Questionnaire. National Center for Research to Improve Postsecondary Teaching and Learning, Ann Arbor, MI
Pintrich, Paul R. (2000). Multiple goals, multiple pathways: The role of goal orientation in learning and achievement. Journal of Educational Psychology, 92(3), 544-555. doi:10.1037/0022-0663.92.3.544

Polson, M. C., y Richardson, J. J. (Eds.). (1988). Foundations of intelligent tutoring systems. Hillsdale, NJ, USA: L. Erlbaum Associates Inc.

Quintana, C., Zhang, M., y Krajcik, J. (2005). A Framework for Supporting Metacognitive Aspects of Online Inquiry Through Software-Based Scaffolding. Educational Psychologist, 40(4), 235-244. doi:10.1207/s15326985ep4004_5

Rodríguez, S., G. Cabanach, R., Piñeiro, I., Valle, A., Núñez, J. C., y González-Pienda, J. A. (2001). Metas de aproximación, metas de evitación y múltiples metas académicas. Psicothema, 13(Número 4), 546-550.

Romero, C., Ventura, S., y García, E. (2008). Data mining in course management systems: Moodle case study and tutorial. Computers and Education, $51(1), 368-384$

Romero, Cristóbal, Ventura, S., Zafra, A., y Bra, P. de. (2009). Applying Web usage mining for personalizing hyperlinks in Web-based adaptive educational systems. Computers \& Education, 53(3), 828 - 840 doi:10.1016/i.compedu.2009.05.003

Ruiz, M. del P. P., Díaz, M. J. F., Soler, F. O., y Pérez, J. R. P. (2008). Adaptation in current e-learning systems. Computer Standards \& Interfaces, 30(1-2), 62-70. doi:10.1016/j.csi.2007.07.006

Sansone, C., Fraughton, T., Zachary, J.L., Butner y J. Heiner, C. (2011) Selfregulation of motivation when learning online: the importance of who, why and how. Education Technology Research Development, 59, 199-212. doi:10.1007/s11423-011-9193-6

Scheiter, K., y Gerjets, P. (2007). Learner Control in Hypermedia Environments. Educational Psychology Review, 19(3), 285-307. doi:10.1007/s10648007-9046-3

Schraw, G., y Moshman, D. (1995). Metacognitive theories. Educational Psychology Review, 7(4), 351-371. doi:10.1007/BF02212307

Schunk, D. H. (1995). Self-efficacy and education and instruction. En Selfefficacy, Adaptation, and Adjustment: Theory, Research, and Application (pp. 281-303). New York, NY, US: Plenum Press.

Schunk, D. H., y Ertmer, P. A. (2000). Self-regulation and academic learning: Self-efficacy enhancing interventions. En M. Boekaerts, P. R. Pintrich, \& M. Zeidner (Eds.), Handbook of Self-regulation (pp. 631-649). San Diego, CA, US: Academic Press.

Shute, V.J., \& Zapata-Rivera, D. (2012). Adaptive educational systems. En P. Durlach y Lesgold, A. M. (Eds.), Adaptive technologies for training and education (pp. 7-27). New York, NY: Cambridge University Press.

Tinajero, C., Castelo, A., Guisande, A., y Páramo, F. (2011). Adaptive teaching and field dependence-independence: instructional implications. Revista Latinoamericana de Psicologia, 43(3), 497-510.

Valle, A., Cabanach, R. G., Núñez, J. C., González-Pienda, J., Rodríguez, S., y Piñeiro, I. (2003). Multiple goals, motivation and academic learning. British Journal of Educational Psychology, 73(1), 71-87. doi:10.1348/000709903762869923

Valle, A., Núñez, J. C., Cabanach, R. G., González-Pienda, J. A., Rodríguez, S., Rosário, P., y Cerezo, R. (2009). Academic goals and learning quality in higher education students. The Spanish journal of psychology, 12(1), 96105.

Valle, A., Rodríguez, S., Cabanach, R. G., Núñez, J. C., González-Pienda, J. A., y Rosário, P. (2010). Motivational Profiles and Differences in Affective, Motivational and Achievement Variables. Universitas Psycbologica, 9(1), 109-121.

Vargas, O. L., Martínez, C. H., y Uribe, Á. C. (2012). Logro de aprendizaje en ambientes hipermediales: andamiaje autorregulador y estilo cognitivo. Revista Latinoamericana de Psicologia, 44(2), 13-26.

Yang, T. C., Hwang, G. J., y Yang, S. J. H. (2013). Development of an Adaptive Learning System with Multiple Perspectives based on Students? Learning Styles and Cognitive Styles. Educational Technology \& Society, 16(4), 185-200.

Zimmerman, B. y Tsikalas, K. (2005). Computer-based learning environments (CBLEs) be used as self- regulatory tools to enhance learning? Educational Psychologist, 40(4), 267-271.

(Artículo recibido: 21-03-2014; revisado: 15-01-2015; aceptado: 18-01-2015) 